\title{
TERAPIA COMUNITÁRIA INTEGRATIVA: UMA PRÁTICA MOBILIZADORA DE AUTOCUIDADO E EDUCAÇÃO EMOCIONAL E SAÚDE INTEGRAL PARA ESTUDANTES DO CURSO DE MEDICINA
}

\author{
TERAPIA COMUNITARIA INTEGRADORA: UNA PRACCTICA MOVILIZADORA DE \\ AUTOCUIDADO Y EDUCACIÓN EMOCIONAL Y SALUD INTEGRAL PARA \\ ESTUDIANTES DE LA CARRERA DE MEDICINA
}

\section{INTEGRATIVE COMMUNITY THERAPY: A MOBILIZING PRACTICE OF SELF- CARE AND EMOTIONAL EDUCATION AND INTEGRAL HEALTH FOR MEDICAL STUDENTS}

\author{
Doralice OTAVIANO ${ }^{1}$ \\ Jurema Valkiria OTAVIANO ${ }^{2}$ \\ Jussara OTAVIANO ${ }^{3}$ \\ Neusa OLIVEIRA ${ }^{4}$
}

RESUMO: O profissional da medicina, vive muitas adaptações durante o percurso de sua formação e após ela. Essas adaptações podem interferir na qualidade de vida e consequentemente na organização de seu processo de trabalho. Perceber as emoções, ajuda na mobilização para criar recursos internos e buscar estratégias de autocuidado. Este estudo, relata a experiência da utilização de uma Prática Integrativa e Complementar; a Terapia Comunitária Integrativa (TCI), criada pelo médico psiquiatra Adalberto Barreto, trata-se de um instrumento de Intervenção Complementar do Ministério da Saúde, utilizada como uma ação coletiva terapêutica, exercida por terapeutas comunitários. Esta técnica tem como objetivo, proporcionar espaços de acolhimento e partilha da vida, onde é possível falar das dores, dos problemas e das potencialidades dos indivíduos e das comunidades. Esse relato de experiência, apresentou um projeto de intervenção terapêutica ofertada para estudantes do primeiro ao sexto ano de um curso de medicina do município de São Paulo. A conclusão da proposta, culminou em resultados positivos no alcance da melhoria da qualidade de saúde integral, mental e emocional deste público.

PALAVRAS-CHAVE: Medicina. Terapia comunitária integrativa. Práticas integrativas e complementares. Cuidado humanizado.

\footnotetext{
${ }^{1}$ Associação Paulista para o Desenvolvimento da Medicina - Hospital São Paulo SPDM/HSP, São Paulo - SP Brasil. Psicóloga no programa Recomeço Família e Coordenadora do curso de Terapia Comunitária no Instituto Afinando Vidas. Doutora em Terapia Familiar pela International Corporate University (I.C.U./CAIFCOM). ORCID: https://orcid.org/0000-0002-6099-6765. E-mail: doramusicoterapia.otaviano@gmail.com

${ }^{2}$ Instituto Afinando Vidas, São Paulo - SP - Brasil. Terapeuta Comunitária. Mestrado em Bioética (USC). ORCID: http://orcid.org/0000-0002-1999-2308. E-mail: arvoregrande2000@yahoo.com.br

${ }^{3}$ Universidade Anhembi Morumbi (UAM), São Paulo - SP - Brasil. Docente do Departamento de Ciências da Saúde. Mestrado em Educação (UNIBAN). ORCID: https://orcid.org/0000-0002-4914-393X. E-mail: jussara.otaviano@gmail.com

${ }^{4}$ Instituto Afinando Vidas, São Paulo - SP - Brasil. Assistente social e Psicóloga Terapeuta de Família e Terapeuta Comunitária. ORCID: https://orcid.org/0000-0003-2625-2161. E-mail: neusadeoliveira@terra.com.br
} 
RESUMEN: El profesional médico, sufre muchas adaptaciones durante el transcurso de su formación y después de ella. Estas adaptaciones pueden interferir en la calidad de vida y, en consecuencia, en la organización de su proceso de trabajo. Percibir emociones, ayuda en la movilización para crear recursos internos y buscar estrategias de autocuidado. Este estudio reporta la experiencia de utilizar una Práctica Integrativa y Complementaria; la Terapia Comunitaria Integrativa (TIC), creada por el psiquiatra Adalberto Barreto, y es un instrumento de Intervención Complementaria del Ministerio de Salud, utilizado como acción terapéutica colectiva, ejercida por terapeutas comunitarios. Esta técnica tiene como objetivo brindar espacios para acoger y compartir la vida, donde se pueda hablar de los dolores, problemas y potencialidades de los individuos y las comunidades. Este relato de experiencia presentó un proyecto de intervención terapéutica, ofrecido a estudiantes del primero al sexto año de un curso de medicina en la ciudad de São Paulo. La conclusión de la propuesta culminó en resultados positivos en la mejora de la calidad de la salud integral, mental y emocional de este público.

PALABRAS CLAVE: Medicina. Terapia comunitaria integradora. Prácticas integradoras y complementarias. Atención humanizada.

ABSTRACT: The medical professional, undergoes many adaptations during the course of formation and after it. These adaptations can interfere in the quality of life and, consequently, in the organization of work process. Perceiving emotions helps in the mobilization to create internal resources and seek self-care strategies. This study reports the experience of using an Integrative and Complementary Practice; the Integrative Community Therapy (ICT), created by the psychiatrist Adalberto Barreto, is an instrument of Complementary Intervention of the Ministry of Health, used as a collective therapeutic action, exercised by community therapists. This technique aims to provide spaces for welcoming and sharing life, where it is possible to talk about the pains, problems and potentials of individuals and communities. This experience report presented a therapeutic intervention project, offered to students from the first to the sixth year of a medical course in the city of São Paulo. The conclusion of the proposal culminated in positive results in improving the quality of comprehensive, mental and emotional health of this public.

KEYWORDS: Medicine. Integrative community therapy. Integrative and complementary practices. Humanized care.

\section{Introdução}

A profissão de medicina, com seu status clássico, tem atravessado a história da humanidade e da ciência com muitas mudanças sociais e tecnológicas. O curso de medicina é hoje um dos mais concorridos do país e exige muita dedicação e compromisso por parte dos estudantes. Este compromisso inicia antes mesmo da inserção na graduação, através de extensivas horas de estudo em cursos pré-vestibulares entre outros.

Para egressos deste curso, apesar da realização do sonho inicial de entrar em uma universidade, existe ao mesmo tempo um cenário repleto de expectativas que cerca este 
momento, mas é também neste espaço, que os acadêmicos se deparam com fatores estressantes que tratam de processos de adaptação e mudanças comportamentais importantes, fatores que caracterizam a saúde integral do estudante de medicina, pois, com saúde plena os estudantes desempenham melhor suas habilidades de aprendizagem (FEODRIPPE et al., 2013).

Neste sentido, instituições de ensino, através de instrumentos específicos como o WHOQOL-bref ${ }^{5}$ que mensuram e avaliam qualidade de vida-QV, vem desenvolvendo pesquisas importantes sobre este tema neste grupo populacional. Pesquisas realizadas na Faculdade de Ciências da Saúde da Universidade Metropolitana de Santos, informam que durante a formação de medicina, a dependência econômica/familiar, associada a não prática de atividade física, a adaptação social restrita, com forte carga de mudança, quando existe deslocamento entre cidades, pode interferir no desempenho escolar (PINCELLI, 2017).

As condições citadas acima, associadas à adaptação social do acadêmico, principalmente à rotina exaustiva de estudos, somado a uma alimentação inadequada, poucos períodos de sono e o esforço para manter a permanência no curso, são características marcantes que levam este público ao estresse físico e emocional, afetando a qualidade de vida dos acadêmicos, expondo estes indivíduos à condições de vulnerabilidade durante o curso de medicina e após sua formação (MEYER et al., 2012).

\section{Proposta de acolhimento humanizado aos estudantes de medicina: implantação e realização}

Ao perceber esse cenário, a coordenação do curso de medicina da Universidade Anhembi Morumbi na cidade de São Paulo no ano de 2016, solicitou a responsável pela disciplina de Saúde Coletiva, uma proposta específica de cuidado à estudantes egressos no curso de medicina, como forma de recepção e acolhimento solidário aos novos alunos.

A proposta tinha como eixo central melhorar a qualidade de vida dos estudantes, além de realizar atividades que pudessem colaborar com sua adaptação (em sua \maioria jovens entre 19 e 25 anos), já que se encontram no ingresso da vida adulta e acadêmica, a intenção desta ação era disparar estratégias que visassem amenizar os efeitos estressantes desses estudantes, com o objetivo de promover um espaço de acolhimento, de construção de vínculos sociais entre

${ }^{5}$ O WHOQOL-bref, é uma versão abreviada do WHOQOL-100, desenvolvido e recomendado pela Organização Mundial da Saúde (OMS) que valoriza a percepção individual, podendo avaliar a QV em diversos grupos e situações, independentemente do nível de escolaridade. O instrumento apresenta propriedades psicométricas satisfatórias e demanda pouco tempo de aplicação. Por meio desse instrumento, é possível descrever a percepção subjetiva de um indivíduo em relação à sua saúde física e psicológica, às relações sociais e ao ambiente em que vive (ALMEIDA-BRASIL et al., 2020). 
alunos, professores e pessoas envolvidas na construção de uma educação emocional saudável, baseada nos conceitos de humanização e práticas integrativas complementares na saúde, entendendo o discente como primeiro usuário destas políticas, além da promoção de redes solidárias e espaços de superação das principais questões que envolvem a adaptação da vida do egresso no mundo acadêmico (BRASIL, 2008).

O método escolhido para a realização desta ação foi a Terapia Comunitária Integrativa (TCI), criada e sistematizada por Adalberto de Paula Barreto, $\mathrm{PhD}$, médico psiquiatra e antropólogo, professor da Universidade Federal do Ceará, que em 1987 criou a TCI em resposta às necessidades de uma determinada população, com foco no atendimento sistemático para grande quantidade de pessoas com problemas emocionais e em sofrimento mental e, também, alinhamento as questões teórico práticas acadêmicas de promoção da saúde mental. Um dos maiores objetivos da TCI é promover atenção primária e coletiva em saúde mental, melhorando a qualidade de vida dos participantes da roda (BARRETO, 2020).

O método utilizado nesta proposta apresentou 3 etapas; primeira etapa, palestra sobre identidade e auto cuidado na formação em medicina com professor Adalberto de Paula Barreto; segunda etapa, realização de rodas de Terapia Comunitária Integrativa para egressos do primeiro ano de medicina; terceira etapa, realização de rodas de terapia comunitária e técnicas de práticas integrativas e complementares do sistema único de saúde para terceiro e quarto ano de medicina.

Quadro - Etapas de implantação da proposta de acolhimento aos estudantes de medicina

\begin{tabular}{|l|l|}
\hline ETAPA 1 & Palestra de sensibilidade e auto cuidado na formação em medicina. \\
\hline ETAPA 2 & Realização de rodas de TCI para egressos do primeiro ano de medicina. \\
\hline ETAPA 3 & $\begin{array}{l}\text { Realização de rodas de TCI associadas a Práticas Integrativas e } \\
\text { Complementares. }\end{array}$ \\
\hline
\end{tabular}

Fonte: elaborado pelos autores

Ressalta-se que boa parte deste público faz parte da geração $\mathrm{Y}^{6}$. Na primeira etapa, estudantes foram contemplados com uma palestra do Professor Adalberto Barreto que iniciou sua fala contando seu percurso pessoal e trajetória de estudos, dando ênfase na contradição que encontrou durante sua formação em medicina, entre o cultural e o acadêmico, e relatou como o processo de formação e de autoconhecimento integrou-se à sua prática.

Duas áreas que parecem ser dissociadas, mas não são, pois não se pode descartar as nossas histórias pessoais de vida, as nossas raízes culturais, em detrimento da vida científica e

6 Geração Y: pessoas nascidas entre o fim dos anos 80 e meados de 2000. Disponível em: http://medimagem.com.br/noticias/quem-e-a-geracao-y,12683. Acesso em: 03 set. 2020. 
da profissão. É importante potencializá-las como recurso para si próprio e para o outro refinando assim nossas capacidades de inteligências intra e interpessoais.

Professor Adalberto também dialogou sobre a necessidade do médico ampliar seus conhecimentos acerca da leitura dos sintomas, já que o ser humano se expressa a partir de vários códigos: linguístico, corporal e espiritual. Destacou que o sintoma tem valor de comunicação e pautou-se num olhar abrangente, complexo e profundo acerca dos sintomas que aparecem para serem diagnosticados, ancorando sua fala em uma visão sistêmica, social e antropológica.

Algumas de suas falas Barreto (2019) demonstram como vê o papel do médico:

[...] Os limites da minha prática, residem nos limites da minha percepção [...] O bom médico sabe acolher com afeto e simplicidade [...] Se preocupem somente em diagnosticar e compreender [...] Como médico, faço o que a medicina pode fazer, mas se eu tenho mais recursos eu faço [...] Somos despertadores de lembranças [...] É preciso estabelecer vínculo com o paciente $[\ldots]$.

Em sua explanação, abriu espaço para o diálogo com os alunos, que participaram tanto com depoimentos pessoais como com perguntas. A participação foi positiva, sendo que vários alunos quiseram conversar com o professor Adalberto Barreto ao término da atividade, dandolhe um retorno sobre a reverberação de sua fala e sobre a necessidade e importância de pensar no cuidado de quem cuida do outro. A atividade possibilitou aos estudantes de medicina ampliar sua concepção de atendimento clínico, da relação/vínculo entre paciente e médico, da importância do autoconhecimento e da saúde mental e bem estar físico de quem cuida.

A segunda etapa caracterizou-se a partir da realização de rodas de terapia comunitária integrativa, inicialmente para 207 egressos no primeiro ano de medicina na disciplina de práticas médicas durante o estágio de Atenção Primária em Saúde em um território onde existe uma Instituição de Longa Permanência de idosos - ILPI, gerenciada pela Secretaria Municipal de Assistência Social da cidade de São Paulo, que abriga cerca de 60 idosos em situação de alta vulnerabilidade e com um escore 3 no grau de dependência da escala da cruz vermelha espanhola.

Esses jovens estudantes de medicina, em sua maioria caracterizados como geração y, acostumados ao excesso de informações, ao tudo pronto e ao imediatismo, pois nasceram junto ao crescimento das telecomunicações e popularização dos computadores, televisões a cabo, internet, novas tecnologias e globalização, são plugados e com rápida velocidade mental, o que leva à instantaneidade no foco e à flexibilidade.

Porém a relação dos alunos com a vida dentro da Instituição de Idosos-ILPI por meio de práticas médicas e terapia comunitária integrativa proporcionou o encontro do coletivo com 
o individual, do idoso com o jovem, dos antigos sentimentos primários com novas percepções de vida, além de grandes imersões nas complexidades da condição humana e diversas possibilidades de fortalecimento e transformação diante da realidade (PINCELLI, 2017).

A reação dos egressos ao chegar no espaço da ILPI, era sempre muito interessante, pois tratava-se de um dos primeiros contatos com uma pessoa altamente debilitada física e/ou mentalmente. Nos primeiros minutos a reação era de perplexidade diante da vulnerabilidade e do abandono, em alguns momentos certo distanciamento e medo diante da situação de sofrimento, mas ao longo dos primeiros minutos esta primeira impressão ia se desfazendo em função do ambiente acolhedor propiciado pelo espaço e pelos próprios idosos residentes.

A atividade foi dividida em dois tempos; aferição de sinais vitais dos idosos e realização da terapia comunitária integrativa para os estudantes. Após a aferição dos sinais vitais e visível envolvimento dos estudantes com o contexto, os mesmos eram encaminhados para outro espaço (o refeitório local), também aberto aos colaboradores do local e residentes da casa, para realização do segundo momento, a terapia comunitária integrativa.

Durante uma hora e meia de terapia comunitária integrativa, os estudantes traziam questões importantes no seu contexto de vida atual, cujos principais temas abordados eram; competição na vida acadêmica, solidão na cidade, falta de autenticidade e falta de cidadania entre as pessoas, tristeza, angustia, medo, luto, pressão em relação as provas e a vida acadêmica, administração do tempo, falta de humanização e sensibilidade com a vulnerabilidade humana. Também apresentaram temas como o uso abusivo do celular impedindo a fruição nas relações, falta de estado de presença.

As rodas sempre finalizavam com experiências de superação, vindas do próprio grupo, diante dos problemas apresentados. E um forte sentimento de solidariedade, interação e construção de vínculos entre o grupo de estudantes. Interessante observar que, ao longo das rodas, alguns idosos passaram a frequentar o ambiente da TCI como ouvintes e criar outros vínculos com os estudantes.

A terceira e última etapa da proposta aconteceu entre os anos de 2016, 2017, 2018, 2019 e 2020 com cerca de 300 alunos envolvidos nas respectivas turmas do terceiro e quarto ano de medicina, em sala de aula, nas disciplinas de saúde coletiva e gestão em serviços de saúde aliadas a temas transversais que se relacionam com as práticas integrativas e complementares do Sistema Único de saúde.

Neste momento, com os estudantes mais adaptados ao ambiente acadêmico e conscientes de seu estado emocional, a proposta da TCI se a apresentava como recurso 
mobilizador para permanência no curso e, principalmente, em momentos de maior pressão com a realização das provas semestrais.

Estudantes relataram que antes da introdução das técnicas de terapia comunitária, cuidando do cuidador e naturologia (meditação, massagem, escalda pés, aromaterapia e musicoterapia), apresentavam sintomas físicos relacionados a pânico, ansiedade, estresse, bem como desconforto respiratório, sudorese intensa, náusea e até vomito nos momentos de testes teóricos e práticos. Estes sintomas após a aplicação da TCI associado às práticas integrativas diminuíam intensamente e ou até desapareciam no decorrer dos semestres (TESSER; SOUSA; NASCIMENTO, 2018).

No primeiro semestre de 2020 em função da pandemia de covid-19, e da mudança de aulas presenciais para as aulas remotas, cerca de 205 de alunos egressos no curso de medicina foram beneficiados com rodas de terapia comunitária integrativa on-line.

\section{O encontro da Terapia Comunitária Integrativa com a educação; uma forma mobilizadora de auto cuidado}

De 2016 até o primeiro semestre de 2020 cerca de 712 estudantes de medicina foram assistidos em rodas de Terapia Comunitária Integrativa. A Terapia Comunitária Integrativa tem pilares importantes, pois relaciona-se com as tecnologias leves e relacionais de Emerson Elias Merry, resgata o conceito da simplicidade na linguagem, em uma dimensão muito profunda e acolhedora quando lida com a complexidade dos princípios da Política Nacional de Humanização (COELHO; JORGE, 2009).

Também encontra-se com as teorias educacionais internacionais importantes, como de Burrhus Frederic Skinner quando trata positivamente da questão comportamental no estimulo resposta na aprendizagem, encontra-se com Jean William Fritz Piaget que estuda as estruturas cognitivas e se aproxima de etapas importantes da terapia comunitária quando menciona que o professor não ensina, mas arranja modos da própria criança descobrir, ou seja, o professor cria situações problema e redimensiona o papel do educando.

Também aproxima-se, de Lev Vygotsky na redução da distância entre o que já se sabe e o que se pode saber, com alguma assistência método caracterizado como a zona de desenvolvimento proximal- ZDP, conectando-se com conceitos de diversidade, e por fim resgata a sabedoria tradicional e coletiva através da teoria educacional, mais brasileira e completa, de Paulo Freire, que apresenta a decodificação do código, buscando significado social 
e clareza do mundo vivido a partir da problematização, o que imprimi uma visão mais crítica do mundo e da capacidade de transformar a realidade. (VIOTTO et al., 2009; FREIRE, 2019)

\section{Considerações finais}

Assim, ligado à conceitos educacionais tão completos, a aplicação da terapia comunitária em ambiente acadêmico delineia um espaço importante de amparo e alívio do estresse emocional vivido por alunos do curso de medicina, além de prevenir doenças mentais importantes como agorafobia, esquizofrenia provocados pela inconstância e fragilidade das relações sociais ou pelo efeito da situação pandêmica vivida na atualidade (ALMEIDA, 2020).

Nesta proposta inovadora, em que desde o primeiro semestre até o último do curso de medicina, o estudante tem contato com Práticas Integrativas e complementares e com a Terapia Comunitária Integrativa.

Essas ações funcionam como fomentadoras de cidadania, de redes sociais solidárias, fortalecimento da identidade cultural das pessoas, além de proporcionar um espaço de afeto, de acolhimento da fala, da livre expressão do sofrimento, das situações de críticas como expressão de conflitos, dúvidas, possibilidades de soluções, sem risco de exclusão dos componentes tratando da valorização da diferença como um referencial positivo de cada um, oferecendo a oportunidade deste grupo social, construir uma rede de interação e resgatar sua da qualidade integral de saúde, vida e autoestima, propiciando mais estabilidade e resiliência aos profissionais da medicina.

Ampliar o acesso da educação médica superior, apresentando a importância do olhar para o autocuidado emocional e integral, também resgata na figura do médico o valor da beneficência durante sua carreira. Universidades que promovem essas práticas também auxiliam na habilidade deste profissional em exercitar a prática da saúde única e do cuidado integral com qualidade para seus usuários. A conclusão desta experiência traz o entendimento que atividades como estas proporcionam um olhar mais humanizado não só para as pessoas que buscam ajuda médica, mas para a própria medicina.

AGRADECIMENTOS: À todos os estudantes de medicina que participaram da proposta, aos médicos; Adalberto de P. Barreto criador da técnica: Terapia Comunitária Integrativa, à Robson R Oliveira e Délio E. M. Filho, coordenadores do curso de medicina da Universidade Anhembi Morumbi, entre os anos de 2015 e 2020, aos médicos terapeutas comunitários formados e em formação no Instituto Afinando Vidas; Simone A.de Oliveira, Leon. H.S. Mourão, Aline. O. 
Laurindo, Edenilza. C.A Mendes, Fernanda. Santana, Renato. C.V. Guimarães e Renan M. Louvatto médico e vizinho sensível que com sua música e o despertar de seu auto cuidado nos ajudou atravessar os dias mais difíceis de isolamento e escrita deste artigo durante a pandemia.

\section{REFERÊNCIAS}

ALMEIDA, H. P. Repercussão do Isolamento social na incidência de transtorno do estresse pós traumático. 2020. Monografia (Trabalho de Conclusão do Curso de Medicina) Universidade Anhembi Morumbi, São Paulo, 2020.

ALMEIDA-BRASIL, C. C. et al. Qualidade de vida e características associadas: aplicação do WHOQOL-BREF no contexto da Atenção Primária à Saúde. Ciênc. saúde coletiva, Rio de Janeiro, v. 22, n. 5, p. 1705-1716, maio 2017. Disponível em: http://www.scielo.br/scielo.php?script=sci_arttext\&pid=S141381232017002501705\&lng=en\&nrm=iso. Acesso em: 4 set. 2020.

BARRETO A. P. Terapia comunitária: passo a passo. 5. ed. Fortaleza: LCR, 2019.

BARRETO, A. et al. Integrative Community Therapy in the Time of the New Coronavirus Pandemic in Brazil and Latin America. World Soc Psychiatry, v. 2, n. 2, p. 103-105, 2020. Disponível em: http://www.worldsocpsychiatry.org/temp/WorldSocPsychiatry221037349955 202459.pdf. Acesso em: 28 set. 2020.

BRASIL. Ministério da Saúde. Secretaria de Atenção à Saúde. Núcleo Técnico da Política Nacional de Humanização. 4. ed. Brasília: Editora do Ministério da Saúde, 72 p., 2008. Disponível em: http://bvsms.saude.gov.br/bvs/publicacoes/cadernos_humanizaSUS.pdf. Acesso em: 4 set. 2020.

COELHO, M. O.; JORGE, M. S. B. Tecnologia das relações como dispositivo do atendimento humanizado na atenção básica à saúde na perspectiva do acesso, do acolhimento e do vínculo. Ciência \& Saúde Coletiva, v. 14, supl. 1, p. 1523-1531, 2009. Disponível em: https://www.scielo.br/pdf/csc/v14s1/a26v14s1.pdf. Acesso em: 15 ago. 2020.

FEODRIPPE, L. A. et al. Qualidade de vida de estudantes de Medicina: uma revisão. Rev. bras. educ. med., v. 37, n. 3, 2013. Disponível em: https://www.scielo.br/pdf/rbem/v37n3/14.pdf. Acesso em: 30 jun. 2020.

FREIRE, N. Dossiê Paulo Freire: O Legado Global. Educação em Revista, Belo Horizonte, V. 35, 2019.

GOLEMAN, D. et al. $\mathbf{O}$ foco triplo: uma nova abordagem para a educação. 1. ed. São Paulo: Objetiva, 2015.

MEYER, C. et al. Qualidade de vida e estresse ocupacional em estudantes de medicina. Revista Brasileira de Educação Médica, v. 36, n. 4, p. 489-498, 2012. Disponível em: https://www.scielo.br/pdf/rbem/v36n4/07. Acesso em: 30 jun. 2020. 
PINCELLI, A. Geração Z e suas respostas comportamentais e emotivas nas redes sociais e virtuais. Orientadora: Adriane Beatriz de Souza Serapião. 2017. 204 f. Dissertação (Mestrado em Desenvolvimento Humano e Tecnologias) - Universidade Estadual Paulista, Rio Claro, SP, 2017. Disponível em:

https://repositorio.unesp.br/bitstream/handle/11449/151376/mussio_rap_me_rcla.pdf?sequenc $\mathrm{e}=3$ \&is Allowed=y.Acesso em: 30 jun. 2020.

SANTOS, J. L. G. et al. Risco e vulnerabilidade nas Práticas dos Profissionais de Saúde. Rev. Gaúcha Enfermagem, v. 33, n. 2, 2012. Disponível em:

https://www.scielo.br/scielo.php?script=sci_arttext\&pid=S1983-14472012000200028. Acesso em: 30 jun. 2020.

TESSER D. C.; SOUSA, I. M. C. de; NASCIMENTO, M. C. do. Práticas Integrativas e Complementares na Atenção Primária à Saúde brasileira. Saúde debate [online], v .42, n. spe. 1, p.174-188, 2018. Disponível em: https://www.scielo.br/pdf/sdeb/v42nspe1/0103-1104sdeb-42-spe01-0174.pdf. Acesso em: 30 jun. 2020.

VIOTTO, I. A. T. et al. As compreensões do humano para Skinner, Piaget, Vygotsky e Wallon: Pequena Introdução às Teorias e suas Implicações na Escola. Psic. da Ed., São Paulo, v. 29, p. 27-55, 2009. Disponível em: http://pablo.deassis.net.br/wpcontent/uploads/As-compreens\%C3\%B5es-do-humano-para-Skinner-Piaget-Vygotski-eWallon.pdf. Acesso em: 30 jun. 2020.

\section{Como referenciar este artigo}

OTAVIANO, D.; OTAVIANO, J. V.; OTAVIANO, J.; OLIVEIRA, N. Terapia Comunitária Integrativa: uma prática mobilizadora de auto cuidado e educação emocional e saúde integral para estudantes do curso de medicina. Temas em Educ. e Saúde, Araraquara, v. 16, n. esp. 1, p. 422-431, set., 2020. e-ISSN 2526-3471. DOI: https://doi.org/10.26673/tes.v16iesp.1.14321

Submetido em: 20/05/2020

Revisões requeridas: $30 / 05 / 2020$

Aprovado em: 25/08/2020

Publicado em: 30/09/2020 